\title{
Anomalous Scaling of Dynamical Large Deviations
}

\author{
Daniel Nickelsen ${ }^{*}$ and Hugo Touchette ${ }^{\dagger}$ \\ National Institute for Theoretical Physics (NITheP), Stellenbosch 7600, South Africa \\ and Institute of Theoretical Physics, Department of Physics, University of Stellenbosch, Stellenbosch 7600, South Africa
}

(Received 16 March 2018; revised manuscript received 18 July 2018; published 31 August 2018)

\begin{abstract}
The typical values and fluctuations of time-integrated observables of nonequilibrium processes driven in steady states are known to be characterized by large deviation functions, generalizing the entropy and free energy to nonequilibrium systems. The definition of these functions involves a scaling limit, similar to the thermodynamic limit, in which the integration time $\tau$ appears linearly, unless the process considered has long-range correlations, in which case $\tau$ is generally replaced by $\tau^{\xi}$ with $\xi \neq 1$. Here, we show that such an anomalous power-law scaling in time of large deviations can also arise without long-range correlations in Markovian processes as simple as the Langevin equation. We describe the mechanism underlying this scaling using path integrals and discuss its physical consequences for more general processes.
\end{abstract}

DOI: 10.1103/PhysRevLett.121.090602

The fluctuations of thermodynamic quantities, such as work, heat, or entropy production, are known to play an important role in the physics of molecular motors, computing devices, and other small systems that function at the nano- to mesoscales in the presence of noise [1-4]. The distribution of these quantities is described in many cases by the theory of large deviations [5] in terms of large deviation functions, which play the role of nonequilibrium potentials similar to the free energy and entropy [6-8]. These functions are important as they characterize the response of nonequilibrium processes to external perturbations [9-11], general symmetries in their fluctuations known as "fluctuation relations" (see Ref. [12] for a review), as well as dynamical phase transitions [13-17].

The definition of large deviation functions involves a limit similar to the thermodynamic limit in which the logarithm of generating functions or probabilities are divided by a scale parameter (e.g., volume, particle number, noise power, or integration time $\tau$ ), which is taken to diverge [7]. This applies, for example, to interacting particle systems, such as the exclusion and zero-range processes, which have been actively studied as microscopic models of energy and particle transport [18-21]. In this case, large deviation functions are defined by taking a large-volume or hydrodynamic limit [22], as well as a limit involving $\tau$ when considering time-integrated or dynamical observables such as the current or activity [19-21].

In this Letter, we show that the latter limit must sometimes be replaced by $\tau^{\xi}$ with $\xi \neq 1$ to obtain well-defined large deviation functions. Such an anomalous scaling of large deviations arises in many stochastic processes, but it is understood (and now widely assumed) to apply to processes that are non-Markovian or involve constraints that lead to long-range correlations. Examples include random collision gases [23], disordered and history-dependent random walks
[24-27], the Wiener sausage [28], tracer dynamics [29-31], the KPZ equation [32-34], and branching processes [35-37]. Our contribution is to show that the same anomalous scaling can arise without long-range correlations and in processes that are Markovian, ergodic, and noncritical. Moreover, we show that the rate function, one of two important large deviation functions, can be nonconvex, which challenges yet another assumption held in large deviation theory and nonequilibrium statistical physics.

These results apply to a large class of processes, as will be argued, but to illustrate them in the simplest way possible, we consider the dynamics of a Brownian particle described by the overdamped Langevin equation or Ornstein-Uhlenbeck process,

$$
\dot{X}_{t}=-\gamma X_{t}+\sigma \eta_{t},
$$

where $X_{t} \in \mathbb{R}$ is the position of the Brownian particle at time $t, \gamma>0$ is the damping, $\eta_{t}$ is a delta-correlated, Gaussian white noise with zero mean, and $\sigma>0$ is the noise intensity, proportional to the square root of the temperature for a thermal environment. For this process, we consider the dynamical observable to be

$$
A_{\tau}=\frac{1}{\tau} \int_{0}^{\tau} X_{t}^{\alpha} d t
$$

where $\alpha$ is an integer assumed to be positive and $\tau$ is again the integration time.

Various versions of this model, determined by $\alpha$, have been considered in the context of nonequilibrium systems and turbulence. The case $\alpha=1$, for instance, is related to Brownian particles pulled by laser tweezers, for which $A_{\tau}$ represents the work (per unit time) done by the laser in the harmonic regime [38]. Alternatively, $X_{t}$ can be interpreted 
as the voltage in a circuit perturbed by Nyquist noise, with $A_{\tau}$ then playing the role of dissipated power [39]. For $\alpha=2, A_{\tau}$ is a statistical estimator of the variance of $X_{t}$, which can be used to measure the damping $\gamma$ or the diffusion constant of Brownian motion $(\gamma=0)$ [40-43]. Finally, the value $\alpha=3$ determines the third moment of $X_{t}$, related in stochastic models of flow velocity fluctuations to the energy rate transferred in the turbulent cascade, while higher moments $(\alpha>3)$ are important for probing smallscale intermittency [44-47].

We are interested here to study the full probability distribution of $A_{\tau}$ denoted by $P_{\tau}(a)$. In the "normal" regime of large deviations, this distribution scales as

$$
P_{\tau}(a) \sim e^{-\tau I(a)}
$$

for large integration times, $\tau \gg 1$, so that the limit

$$
I(a)=\lim _{\tau \rightarrow \infty}-\frac{1}{\tau} \ln P_{\tau}(a)
$$

exists and defines a nontrivial function called the rate function [5]. This function is positive and such that $I\left(a^{*}\right)=0$ for the expected value

$$
a^{*}=\int_{-\infty}^{\infty} \rho_{s}(x) x^{\alpha} d x
$$

obtained from the stationary distribution $\rho_{s}(x)$ of $X_{t}$. This implies that fluctuations away from $a^{*}$ are exponentially unlikely, so that $A_{\tau} \rightarrow a^{*}$ with probability 1 as $\tau \rightarrow \infty$, in accordance with the ergodic theorem. In this limit, $I(a)$ thus characterizes the likelihood of fluctuations of $A_{\tau}$ around $a^{*}$, in the same way that the entropy characterizes the fluctuations of equilibrium systems around their equilibrium state in the thermodynamic limit (see Ref. [7] for more details on this analogy).

Normal large deviations are found when $\alpha=1$ or $\alpha=2$, and in both cases the rate function is obtained from the dominant eigenvalue of the Feynman-Kac equation for the generating function of $A_{\tau}$. This spectral result is well known [48-50]: it is detailed in Ref. [51] and is briefly summarized in the Supplemental Material for completeness [52]. The end result is that $I(a)$ is given by a Legendre transform of what is essentially the ground state energy of the quantum harmonic oscillator. From this mapping, one finds a parabolic rate function associated with Gaussian fluctuations of $A_{\tau}$ for $\alpha=1$, and a more complicated rate function describing non-Gaussian fluctuations for $\alpha=2$ [56].

A problem arises, however, when $\alpha>2$. Then the mapping yields a quantum potential which is not confining and, therefore, has no ground state energy for some parameter values. For $\alpha=3$, for example, one finds that the quantum potential is

$$
V_{k}(x)=\frac{\gamma^{2} x^{2}}{2 \sigma^{2}}-\frac{\gamma}{2}-k x^{3},
$$

where $k$ is the real parameter entering in the generating function of $A_{\tau}$, which is related to the rate function by Legendre transform (see the Supplemental Material [52]). This potential has no finite ground state energy for any $k \in \mathbb{R}$ because of the $x^{3}$ term, which means that the rate function is not related to a ground state energy or dominant eigenvalue. The same applies for any odd integers $\alpha>3$, suggesting that $P_{\tau}(a)$ either does not scale exponentially with $\tau$ or that the scaling is exponential but becomes anomalous, in the sense that

$$
P_{\tau}(a) \sim e^{-\tau^{\xi} I(a)}
$$

with $\xi \neq 1$, and so that $\tau$ must be replaced by $\tau^{\xi}$ in the limit of Eq. (4) to obtain the correct rate function.

There is no method, as far as we know, that can give the rate function of $A_{\tau}$ in this new scaling regime for arbitrary noise amplitude [57]. However, we can explore the form of $P_{\tau}(a)$ in the low-noise limit using the well-known saddlepoint, instanton, or optimal path approximation method, widely used to study noise-activated transition phenomena in equilibrium and nonequilibrium systems [58-63], including the KPZ equation [64-66] and interacting particle systems described in the hydrodynamic limit by stochastic transport equations [19-21]. This approximation is summarized in the Supplemental Material [52] and leads here to

$$
P_{\tau}(a) \sim e^{-\mathcal{S}_{\tau}[\bar{x}]}
$$

as $\sigma \rightarrow 0$, where $\bar{x}(t)$ is the optimal path or instanton that minimizes the action

$$
\mathcal{S}_{\tau}[x]=\frac{1}{2 \sigma^{2}} \int_{0}^{\tau}[\dot{x}(t)+\gamma x(t)]^{2} d t
$$

of the Ornstein-Uhlenbeck process subject to the constraint $A_{\tau}=a$ in Eq. (2). In our case, $\bar{x}(t)$ is given by the following Euler-Lagrange equation:

$$
\ddot{x}(t)=\gamma^{2} x(t)-\beta \sigma^{2} \alpha x(t)^{\alpha-1}
$$

with free boundary conditions, where $\beta$ is a Lagrange parameter that fixes the constraint $A_{\tau}=a$. Equivalently, we can obtain $\bar{x}(t)$ by solving Hamilton's equations associated with the Hamiltonian,

$$
H(x, p)=\frac{\sigma^{2} p^{2}}{2}-\gamma x p+\beta x^{\alpha} .
$$

We cannot solve these equations exactly for finite $\tau$ and $\alpha>2$. However, we find numerically that, as $\tau \rightarrow \infty$, $\bar{x}(0)$ and $\bar{x}(\tau)$ approach 0 , implying that the associated momentum $p=(\dot{x}+\gamma x) / \sigma^{2}$ and "energy" $H$ also vanish. The infinite-time instanton thus evolves in phase space on 


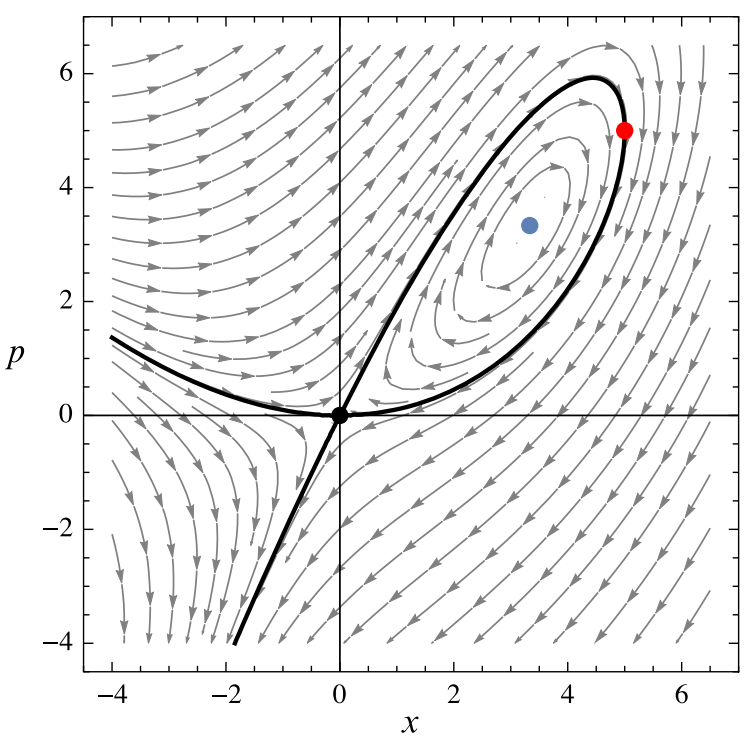

FIG. 1. Stream vector field of Hamilton's equations describing the instanton in phase space for $\alpha=3, \gamma=1, \sigma=1$, and $\beta=0.1$. Black line: $H(x, p)=0$ manifold. Black point: unstable fixed point at the origin. Blue point: stable fixed point $\left(x^{*}, p^{*}\right)$. Red point: turning point $(\hat{x}, p(\hat{x}))$.

the $H=0$ manifold, as shown in Fig. 1: it escapes the unstable origin, performs a loop on the positive part of the zero-energy manifold in finite time, before returning to $(0,0)$. As a result, we can express the action as

$$
\mathcal{S}_{\tau}[\bar{x}]=\oint_{H=0} p d x+\beta \tau a .
$$

The line integral can be calculated exactly and so can the Lagrange parameter as a function of the constraint $A_{\tau}=a$ (see the Supplemental Material [52]). Combining these, we find that $\mathcal{S}_{\tau}[\bar{x}]$ is proportional to $\tau^{2 / \alpha}$, so that $P_{\tau}(a)$ has the form of Eq. (7) with $\xi=2 / \alpha$, and

$$
I(a)=\frac{\mathcal{S}_{\tau}[\bar{x}]}{\tau^{\xi}}=c \frac{\gamma^{[(\alpha+2) / \alpha]}}{\sigma^{2}} a^{(2 / \alpha)},
$$

where

$$
\begin{aligned}
c= & \pi^{[(\alpha-2) /(2 \alpha)]}\left(\frac{2}{\alpha+2} \frac{\Gamma\left(\frac{2}{\alpha-2}\right)}{\Gamma\left(\frac{\alpha+2}{2 \alpha-4}\right)}+\frac{1}{\alpha-2} \frac{\Gamma\left(\frac{\alpha}{\alpha-2}\right)}{\Gamma\left(\frac{3 \alpha-2}{2 \alpha-4}\right)}\right) \\
& \times\left(\frac{\alpha-2}{2} \frac{\Gamma\left(\frac{3 \alpha-2}{2 \alpha-4}\right)}{\Gamma\left(\frac{\alpha}{\alpha-2}\right)}\right)^{(2 / \alpha)}
\end{aligned}
$$

is a constant prefactor. In particular,

$$
\begin{aligned}
& I(a)=\left(\frac{9}{10}\right)^{(1 / 3)} \frac{\gamma^{(5 / 3)}}{\sigma^{2}} a^{(2 / 3)} \\
& I(a)=\left(\frac{4}{3}\right)^{(1 / 2)} \frac{\gamma^{(3 / 2)}}{\sigma^{2}} a^{(1 / 2)}
\end{aligned}
$$

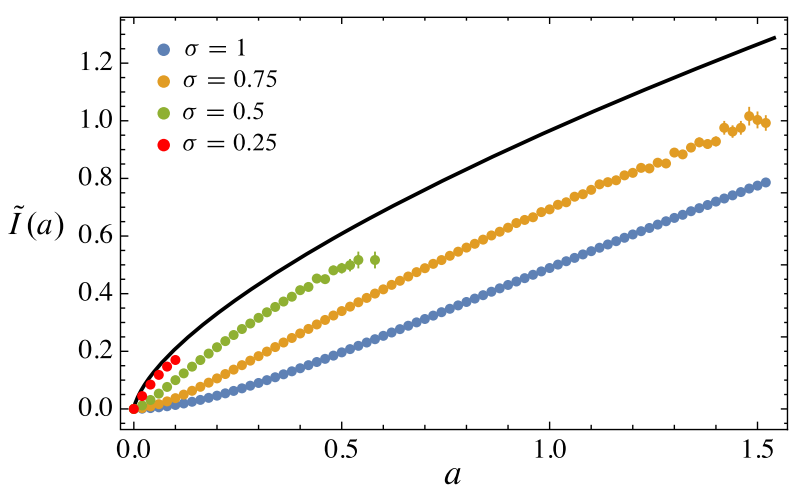

FIG. 2. Scaled rate function $\tilde{I}(a)=\sigma^{2} I(a)$ for $\gamma=1$ and $\alpha=3$, plotted for $a \geq 0$. Black curve: low-noise result of Eq. (13), which is independent of $\sigma$ after rescaling. Data points: Monte Carlo results for different noise amplitudes. Error bars are shown on all points but are in most cases too small to be seen (see text and the Supplemental Material [52]).

for $\alpha=3$ and $\alpha=4$, respectively. Note that, for simplicity, we only give the result for $a \geq 0$, since $A_{\tau} \geq 0$ when $\alpha$ is even, whereas $I(-a)=I(a)$ when $\alpha$ is odd due to the symmetry of the process.

This exact expression for the rate function is our main result. Although it is valid in the limit $\sigma \rightarrow 0$, we show in Fig. 2 that it gives a good approximation of the "true" rate function obtained by Monte Carlo simulations for $\sigma>0$, up to around $\sigma=0.5$. To obtain this plot, we simulated $10^{9}$ paths of the Ornstein-Uhlenbeck process using the EulerMaruyama scheme and transformed the histogram of $A_{\tau}$ for different $\tau$ according to the large deviation limit of Eq. (4) with $\tau$ replaced by $\tau^{\xi}$, so as to get an estimate of $I(a)$ (see the Supplemental Material [52]). We also plot $\tilde{I}(a)=$ $\sigma^{2} I(a)$ rather than $I(a)$, since the low-noise prediction of Eq. (13) is independent of $\sigma$ under this rescaling.

The results are found to converge for $\tau \gtrsim 20$ or $\tau \gtrsim 30$, depending on the noise amplitude considered, and confirm that $P_{\tau}(a)$ scales anomalously according to Eq. (7) with the predicted $\xi=\alpha / 2$. There are very few data points for $\sigma=0.25$, since we are dealing with rare fluctuations that are suppressed exponentially in $T$ and $1 / \sigma^{2}$, but those obtained confirm the function obtained in Eq. (13), which is, interestingly, nonconvex and homogenous (or scale free). The $\tau$ scaling with $\xi=2 / \alpha$ is consistent with the fact that there is no mapping to the quantum problem, since it implies that the generating function of $A_{\tau}$ diverges for all $k \neq 0$. This can also be seen by noting that, since $\xi<1$ for $\alpha>2$, we get $I(a)=0$ if we use the "wrong" limit shown in Eq. (4). The Legendre transform of that zero rate function diverges for all nonzero values of the conjugate parameter $k$, which is what the quantum problem predicts in the absence of bound states (see the Supplemental Material [52]).

This applies to any odd $\alpha>2$, for which the mean $a^{*}$, as given by Eq. (5), vanishes since $\rho_{s}(x)$ is even in $x$. For even 


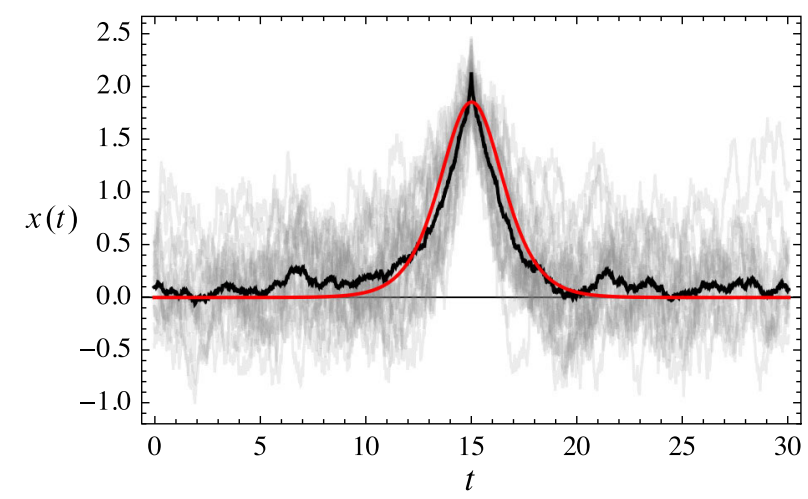

FIG. 3. Typical paths of the process (in gray) satisfying the constraint $A_{T}=a$ found by direct Monte Carlo simulations, compared with the instanton (in red) computed numerically. Parameters: $\gamma=1, \sigma=0.5, \tau=30$, and $a=0.45 \pm 0.02$. The maximum of each instanton is translated to $t=15$. Black curve: average instanton.

values of $\alpha>2$, the situation is slightly more involved. Then $A_{T} \geq 0$ and, for $0 \leq a<a^{*}, A_{T}$ has normal large deviations with $\xi=1[67,68]$, since the quantum problem has a bound state, from which we can obtain the exact rate function, as described in the Supplemental Material [52]. For $a>a^{*}$, however, we have anomalous large deviations with $\xi=2 / \alpha$ and a rate function given, in the low-noise limit, by our general result of Eq. (13), which predicts that the mean is 0 , consistently with the fact that $a^{*} \rightarrow 0$ as $\sigma \rightarrow 0$.

To illustrate the physical meaning of the instanton, we show in Fig. 3 typical paths of the process with $\gamma=1$ and $\sigma=0.5$ leading to a given fluctuation $A_{\tau}=a$ after $\tau=30$, the observed convergence time. For these parameters, we found 28 out of $10^{9}$ simulated paths reaching the value $A_{\tau}=0.45 \pm 0.02$, which lies on the green curve in Fig. 2 . Since fluctuations can happen in simulations anywhere in the whole time interval $[0, \tau]$, we compare these paths by translating their maximum at the time $\tau / 2$ where the instanton has its own maximum. This also allows us to compute an average fluctuation path which can be compared with the predicted instanton [63].

All the paths are in good agreement, as can be seen, which shows that the low-noise theory correctly predicts how fluctuations are created dynamically by escaping to a position $\hat{x}$, which scales like $(a \tau)^{1 / \alpha}$, over a finite time proportional to $1 / \gamma$. It can be verified (see the Supplemental Material [52]) that approximating this escape path from $x=0$ by two exponentials with rate $\gamma$ reproduces the correct $\tau$ scaling of the action, though not the exact, lownoise expression of the rate function. Similar results are obtained for other values of $A_{\tau}$ and $\alpha>2$, provided that $\tau$ is large enough and $\sigma$ is small enough.

The instanton that we find is similar to those arising in the Kramers escape problem [58], underlying many noise-induced transition phenomena [69]. The essential difference is that we consider a "global" constraint $A_{\tau}=a$ rather than a "local" constraint for the escape that a process reach a given point or set in time. The instanton is also related to condensation phase transitions in interacting particle systems, such as the zero-range process, in which an extensive number of particles accumulate on a spatial site [70-72]. Here, we find "temporal condensates" in the form of trajectories for the fluctuations of $A_{\tau}$ that are localized in time compared to $\tau$ and whose height scales with $\tau$. A related condensation was reported recently in the context of sums of random variables, which can be dominated in some cases by a single, extensive or "giant" value [73-78].

The results that we have presented show that temporal condensation phenomena can arise in simple continuoustime processes and are not necessarily associated with power-law distributions, as found in Refs. [75-77]. They also show, more remarkably, that anomalous large deviations can arise without long-range correlations, non-Markovian dynamics or disorder, and can be linked generally to a breakdown of the quantum formalism used to calculate rate functions. As such, they are expected to arise in other reversible systems for which this formalism can be applied whenever the quantum potential related to the process and observable [51] does not have a finite ground state.

The problem remains to find the exact rate function of $A_{\tau}$ in the anomalous regime for arbitrary noise amplitudes. Most analytical methods rely on the normal scaling of large deviations and, as a result, cannot be applied. This includes the quantum mapping, as mentioned, but also the so-called contraction principle [5]. There is a possibility that one can obtain $I(a)$ by finding the exact generating function of $A_{\tau}$ via, for example, a time-dependent Feynman-Kac equation [42] in which $k$ is scaled with time. However, if $I(a)$ is nonconvex, then even this method will not work, since the Legendre connection between generating functions and rate functions is lost [7].

The same limitations apply to numerical methods developed recently to compute rate functions efficiently. Except for the direct Monte Carlo method used here, all methods, including cloning [79-81] and importance sampling [82-84], work by reweighting trajectories exponentially with time in a normal way. In this sense, the model proposed here should serve as an ideal toy model to develop new analytical and numerical methods that are applicable to physical systems with anomalous large deviations, including the many non-Markovian and disordered processes mentioned in the introduction.

We are grateful to S. Sanbhapandit, S. Majumdar, R. Chetrite, J. Meibohm, and A. Krajenbrink for useful discussions, and also thank M. Kastner for computer access. Support was received from NITheP (postdoctoral fellowship) and the National Research Foundation of South Africa (Grant No. 96199). Additional computations were performed using Stellenbosch University's HPC1. 
*danielnickelsen@sun.ac.za

†htouchet@alum.mit.edu, htouchette@sun.ac.za

[1] F. Ritort, Nonequilibrium Fluctuations in Small Systems: From Physics to Biology, Advances in Chemical Physics Vol. 137 (John Wiley, New York, 2008), pp. 31-123.

[2] K. Sekimoto, Stochastic Energetics, Lecture Notes in Physics, Vol. 799 (Springer, New York, 2010).

[3] C. Jarzynski, Equalities and inequalities: Irreversibility and the second law of thermodynamics at the nanoscale, Annu. Rev. Condens. Matter Phys. 2, 329 (2011).

[4] U. Seifert, Stochastic thermodynamics, fluctuation theorems and molecular machines, Rep. Prog. Phys. 75, 126001 (2012).

[5] A. Dembo and O. Zeitouni, Large Deviations Techniques and Applications, 2nd ed. (Springer, New York, 1998).

[6] Y. Oono, Large deviation and statistical physics, Prog. Theor. Phys. Suppl. 99, 165 (1989).

[7] H. Touchette, The large deviation approach to statistical mechanics, Phys. Rep. 478, 1 (2009).

[8] R. J. Harris and H. Touchette, Large deviation approach to nonequilibrium systems, in Nonequilibrium Statistical Physics of Small Systems: Fluctuation Relations and Beyond, Reviews of Nonlinear Dynamics and Complexity, Vol. 6, edited by R. Klages, W. Just, and C. Jarzynski (Wiley-VCH, Weinheim, 2013), pp. 335-360.

[9] M. Baiesi, C. Maes, and B. Wynants, Fluctuations and Response of Nonequilibrium States, Phys. Rev. Lett. 103, 010602 (2009).

[10] M. Baiesi, C. Maes, and B. Wynants, Nonequilibrium linear response for Markov dynamics I: Jump processes and overdamped diffusions, J. Stat. Phys. 137, 1094 (2009).

[11] C. Maes, K. Netočný, and B. Wynants, Monotonic Return to Steady Nonequilibrium, Phys. Rev. Lett. 107, 010601 (2011).

[12] R. J. Harris and G. M. Schütz, Fluctuation theorems for stochastic dynamics, J. Stat. Mech. (2007) P07020.

[13] J. P. Garrahan, R. L. Jack, V. Lecomte, E. Pitard, K. van Duijvendijk, and F. van Wijland, Dynamical First-Order Phase Transition in Kinetically Constrained Models of Glasses, Phys. Rev. Lett. 98, 195702 (2007).

[14] D. Chandler and J. P. Garrahan, Dynamics on the way to forming glass: Bubbles in space-time, Annu. Rev. Phys. Chem. 61, 191 (2010).

[15] P. I. Hurtado and P. L. Garrido, Spontaneous Symmetry Breaking at the Fluctuating Level, Phys. Rev. Lett. 107, 180601 (2011).

[16] Y. Baek and Y. Kafri, Singularities in large deviation functions, J. Stat. Mech. (2015) P08026.

[17] Y. Baek, Y. Kafri, and V. Lecomte, Dynamical Symmetry Breaking and Phase Transitions in Driven Diffusive Systems, Phys. Rev. Lett. 118, 030604 (2017).

[18] H. Spohn, Large Scale Dynamics of Interacting Particles (Springer Verlag, Berlin, 1991).

[19] B. Derrida, Non-equilibrium steady states: Fluctuations and large deviations of the density and of the current, J. Stat. Mech. (2007) P07023.

[20] L. Bertini, A. De Sole, D. Gabrielli, G. Jona-Lasinio, and C. Landim, Stochastic interacting particle systems out of equilibrium, J. Stat. Mech. (2007) P07014.
[21] L. Bertini, A. De Sole, D. Gabrielli, G. Jona-Lasinio, and C. Landim, Macroscopic fluctuation theory, Rev. Mod. Phys. 87, 593 (2015).

[22] The hydrodynamic limit is equivalent, via the macroscopic fluctuation theory [21], to a low-noise limit.

[23] G. Gradenigo, A. Sarracino, A. Puglisi, and H. Touchette, Fluctuation relations without uniform large deviations, J. Phys. A 46, 335002 (2013).

[24] A. Dembo, Y. Peres, and O. Zeitouni, Tail estimates for one-dimensional random walk in random environment, Commun. Math. Phys. 181, 667 (1996).

[25] N. Gantert and O. Zeitouni, Quenched sub-exponential tail estimates for one-dimensional random walk in random environment, Commun. Math. Phys. 194, 177 (1998).

[26] O. Zeitouni, Random walks in random environments, J. Phys. A 39, R433 (2006).

[27] R. J. Harris and H. Touchette, Current fluctuations in stochastic systems with long-range memory, J. Phys. A 42, 342001 (2009).

[28] M. van den Berg, E. Bolthausen, and F. den Hollander, Moderate deviations for the volume of the Wiener sausage, Ann. Math. 153, 355 (2001).

[29] P. L. Krapivsky, K. Mallick, and T. Sadhu, Large Deviations in Single-File Diffusion, Phys. Rev. Lett. 113, 078101 (2014).

[30] T. Sadhu and B. Derrida, Large deviation function of a tracer position in single file diffusion, J. Stat. Mech. (2015) P09008.

[31] T. Imamura, K. Mallick, and T. Sasamoto, Large Deviations of a Tracer in the Symmetric Exclusion Process, Phys. Rev. Lett. 118, 160601 (2017).

[32] P. Le Doussal, S. N. Majumdar, and G. Schehr, Large deviations for the height in 1D Kardar-Parisi-Zhang growth at late times, Europhys. Lett. 113, 60004 (2016).

[33] P. Sasorov, B. Meerson, and S. Prolhac, Large deviations of surface height in the $1+1$-dimensional Kardar-ParisiZhang equation: Exact long-time results for $\lambda H<0$, J. Stat. Mech. (2017) 063203.

[34] I. Corwin, P. Ghosal, A. Krajenbrink, P. Le Doussal, and L.-C. Tsai, Coulomb-Gas Electrostatics Controls Large Fluctuations of the KPZ Equation, Phys. Rev. Lett. 121, 060201 (2018).

[35] J. T. Cox and D. Griffeath, Occupation times for critical branching Brownian motions, Ann. Probab. 13, 1108 (1985).

[36] O. Louidor and W. Perkins, Large deviations for the empirical distribution in the branching random walk, Electron. J. Probab. 20, 18 (2015).

[37] B. Derrida and Z. Shi, Slower deviations of the branching Brownian motion and of branching random walks, J. Phys. A 50, 344001 (2017).

[38] R. van Zon and E. G. D. Cohen, Stationary and transient work-fluctuation theorems for a dragged Brownian particle, Phys. Rev. E 67, 046102 (2003).

[39] R. van Zon, S. Ciliberto, and E. G. D. Cohen, Power and Heat Fluctuation Theorems for Electric Circuits, Phys. Rev. Lett. 92, 130601 (2004).

[40] D. Florens-Landais and H. Pham, Large deviations in estimation of an Ornstein-Uhlenbeck model, J. Appl. Probab. 36, 60 (1999). 
[41] B. Bercu and A. Rouault, Sharp large deviations for the Ornstein-Uhlenbeck process, Theory Probab. Appl. 46, 1 (2002).

[42] D. Boyer and D. S. Dean, On the distribution of estimators of diffusion constants for Brownian motion, J. Phys. A 44, 335003 (2011).

[43] D. Boyer, D. S. Dean, C. Mejía-Monasterio, and G. Oshanin, Optimal estimates of the diffusion coefficient of a single Brownian trajectory, Phys. Rev. E 85, 031136 (2012).

[44] G. Pedrizzetti and E. A. Novikov, On Markov modelling of turbulence, J. Fluid Mech. 280, 69 (1994).

[45] K. R. Sreenivasan and R. A. Antonia, The phenomenology of small-scale turbulence, Annu. Rev. Fluid Mech. 29, 435 (1997).

[46] T. Matsumoto and M. Takaoka, Large-scale lognormality in turbulence modeled by the Ornstein-Uhlenbeck process, Phys. Rev. E 87, 013008 (2013).

[47] D. Nickelsen, Master equation for She-Leveque scaling and its classification in terms of other Markov models of developed turbulence, J. Stat. Mech. (2017) 073209.

[48] G. L. Eyink, Action principle in nonequilibrium statistical dynamics, Phys. Rev. E 54, 3419 (1996).

[49] S. N. Majumdar and A. J. Bray, Large-deviation functions for nonlinear functionals of a Gaussian stationary Markov process, Phys. Rev. E 65, 051112 (2002).

[50] H. Fujisaka and T. Yamada, Level dynamics approach to the large deviation statistical characteristic function, Phys. Rev. E 75, 031116 (2007).

[51] H. Touchette, Introduction to dynamical large deviations of Markov processes, Physica (Amsterdam) 504A, 5 (2018).

[52] See Supplemental Material at http://link.aps.org/ supplemental/10.1103/PhysRevLett.121.090602 for the effect of boundary terms in normal large deviations, including Refs. [53-55].

[53] R. van Zon and E. G. D. Cohen, Extension of the Fluctuation Theorem, Phys. Rev. Lett. 91, 110601 (2003).

[54] S. Sabhapandit, Work fluctuations for a harmonic oscillator driven by an external random force, Europhys. Lett. 96, 20005 (2011).

[55] S. Sabhapandit, Heat and work fluctuations for a harmonic oscillator, Phys. Rev. E 85, 021108 (2012).

[56] W. Bryc and A. Dembo, Large deviations for quadratic functionals of Gaussian processes, J. Theor. Probab. 10, 307 (1997).

[57] In many of the applications mentioned in the introduction, including the KPZ equation, the large deviation function is obtained in the anomalous scaling via exact representations or mappings based, for example, on Coulomb gases and random matrices. We know of no such mappings for our problem.

[58] L. Onsager and S. Machlup, Fluctuations and irreversible processes, Phys. Rev. 91, 1505 (1953).

[59] M. I. Freidlin and A. D. Wentzell, Random Perturbations of Dynamical Systems, Vol. 260 Grundlehren der Mathematischen Wissenschaften (Springer, New York, 1984).

[60] R. Graham, Macroscopic potentials, bifurcations and noise in dissipative systems, in Noise in Nonlinear Dynamical Systems, edited by F. Moss and P. V.E. McClintock, Vol. 1 (Cambridge University Press, Cambridge, 1989), pp. 225-278.
[61] D. G. Luchinsky, P. V. E. McClintock, and M. I. Dykman, Analogue studies of nonlinear systems, Rep. Prog. Phys. 61, 889 (1998).

[62] D. Nickelsen and A. Engel, Asymptotics of work distributions: The pre-exponential factor, Eur. Phys. J. B 82, 207 (2011).

[63] T. Grafke, R. Grauer, and T. Schäfer, The instanton method and its numerical implementation in fluid mechanics, J. Phys. A 48, 333001 (2015).

[64] I. V. Kolokolov and S. E. Korshunov, Universal and nonuniversal tails of distribution functions in the directed polymer and Kardar-Parisi-Zhang problems, Phys. Rev. B 78, 024206 (2008).

[65] H. C. Fogedby and W. Ren, Minimum action method for the Kardar-Parisi-Zhang equation, Phys. Rev. E 80, 041116 (2009).

[66] B. Meerson, E. Katzav, and A. Vilenkin, Large Deviations of Surface Height in the Kardar-Parisi-Zhang Equation, Phys. Rev. Lett. 116, 070601 (2016).

[67] V. Fatalov, Exact asymptotics of large deviations of stationary Ornstein-Uhlenbeck processes for $L^{p}$-functionals, $p>0$, Probl. Inf. Transm. 42, 46 (2006).

[68] V. Fatalov, Occupation time and exact asymptotics of distributions of $L^{p}$-functionals of the Ornstein-Uhlenbeck processes, $p>0$, Theory Probab. Appl. 53, 13 (2009).

[69] C. W. Gardiner, Handbook of Stochastic Methods for Physics, Chemistry and the Natural Sciences, 2nd ed., Springer Series in Synergetics Vol. 13 (Springer, New York, 1985).

[70] S. Grosskinsky, G. M. Schütz, and H. Spohn, Condensation in the zero range process: Stationary and dynamical properties, J. Stat. Phys. 113, 389 (2003).

[71] R. J. Harris, V. Popkov, and G. M. Schütz, Dynamics of instantaneous condensation in the ZRP conditioned on an atypical current, Entropy 15, 5065 (2013).

[72] P. Chleboun and S. Grosskinsky, Condensation in stochastic particle systems with stationary product measures, J. Stat. Phys. 154, 432 (2014).

[73] J. Szavits-Nossan, M. R. Evans, and S. N. Majumdar, Constraint-Driven Condensation in Large Fluctuations of Linear Statistics, Phys. Rev. Lett. 112, 020602 (2014).

[74] J. Szavits-Nossan, M. R. Evans, and S. N. Majumdar, Condensation transition in joint large deviations of linear statistics, J. Phys. A 47, 455004 (2014).

[75] M. Zannetti, F. Corberi, and G. Gonnella, Condensation of fluctuations in and out of equilibrium, Phys. Rev. E 90, 012143 (2014).

[76] F. Corberi, Large deviations, condensation and giant response in a statistical system, J. Phys. A 48, 465003 (2015).

[77] F. Corberi, Development and regression of a large fluctuation, Phys. Rev. E 95, 032136 (2017).

[78] J. Szavits-Nossan and M. R. Evans, Inequivalence of nonequilibrium path ensembles: The example of stochastic bridges, J. Stat. Mech. (2015) P12008.

[79] C. Giardina, J. Kurchan, and L. Peliti, Direct Evaluation of Large-Deviation Functions, Phys. Rev. Lett. 96, 120603 (2006).

[80] V. Lecomte and J. Tailleur, A numerical approach to large deviations in continuous time, J. Stat. Mech. (2007) P03004. 
[81] C. Giardina, J. Kurchan, V. Lecomte, and J. Tailleur, Simulating rare events in dynamical processes, J. Stat. Phys. 145, 787 (2011).

[82] J. A. Bucklew, Introduction to Rare Event Simulation (Springer, New York, 2004).

[83] H. Touchette, A basic introduction to large deviations: Theory, applications, simulations, in Modern Computational Science
11: Lecture Notes from the 3rd International Oldenburg Summer School, edited by R. Leidl and A. K. Hartmann (BIS-Verlag der Carl von Ossietzky Universität Oldenburg, Oldenburg, 2011).

[84] R. Chetrite and H. Touchette, Variational and optimal control representations of conditioned and driven processes, J. Stat. Mech. (2015) P12001. 